\title{
Crossings and Nestings in Tangled Diagrams
}

\author{
William Y. C. Chen ${ }^{1}$, Jing Qin² and Christian M. Reidys ${ }^{3}$ \\ Center for Combinatorics, LPMC-TJKLC \\ Nankai University, \\ Tianjin 300071, P. R. China \\ ${ }^{1}$ chen@nankai.edu.cn, ${ }^{2}$ qj@cfc.nankai.edu.cn, ${ }^{3}$ duck@santafe.edu
}

Submitted: Oct 25, 2007; Accepted: Jun 20, 2008; Published: Jun 25, 2008

Mathematics Subject Classification: 05A18

\begin{abstract}
A tangled diagram on $[n]=\{1, \ldots, n\}$ is a labeled graph for which each vertex has degree at most two. The vertices are arranged in increasing order on a horizontal line and the arcs are drawn in the upper halfplane with a particular notion of crossings and nestings. Generalizing the construction of Chen et al., we give a bijection between generalized vacillating tableaux with less than $k$ rows and $k$ noncrossing tangled diagrams. We show that the numbers of $k$-noncrossing and $k$-nonnesting tangled diagrams are equal and we enumerate $k$-noncrossing tangled diagrams. Finally, we show that braids, a special class of tangled diagrams, facilitate a bijection between 2-regular $k$-noncrossing partitions and $k$-noncrossing enhanced partitions.
\end{abstract}

\section{Introduction}

In this paper, we study $k$-noncrossing tangled diagrams by generalizing the concept of vacillating tableaux introduced by Chen et al. [3] for $k$-noncrossing partitions and matchings. A set partition (partition) gives rise to an edge set obtained by connecting the elements in each block in numerical order. The latter is called the standard representation of a partition. A partition is called $k$-noncrossing if there does not exist any $k$ $\operatorname{arcs}\left(i_{1}, j_{1}\right),\left(i_{2}, j_{2}\right), \ldots,\left(i_{k}, j_{k}\right)$ such that $i_{1}<i_{2}<\cdots<i_{k}<j_{1}<j_{2}<\cdots<j_{k}$. A $k$-noncrossing matching is defined accordingly, since a matching is simply a partition in which all blocks have sizes two. We first discuss tangled diagrams in relation to partitions and matchings and put our results into this context. Second, we give some background on tangled diagrams. A tangled diagram on $[n]$ is a labeled graph on the vertices $1, \ldots, n$, 
drawn in increasing order on a horizontal line. The arcs are drawn in the upper halfplane. In general, a tangled diagram has isolated points and the following types of arcs:

(n)

For instance,
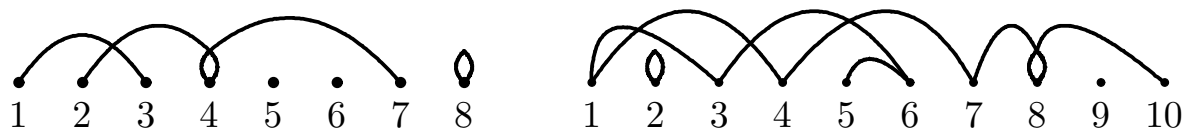

are two tangled diagrams. Tangled diagrams in which all vertices of degree two, $j$, are either incident to loops $(j, j)$, or crossing $\operatorname{arcs}(i, j)$ and $(j, h)$, where $i<j<h$, are called braids. For example, the first tangled diagram of (1.1) is a 3-noncrossing braid. A matching on $[2 n]=\{1,2, \ldots, 2 n\}$ is a 1-regular tangled diagram. For instance, the matching $\{(1,8),(2,6),(3,10),(4,5),(7,9)\}$ is the first tangled diagram in (1.2). The standard representation of a partition is a tangled diagram. For example, the tangled diagram induced by $\pi=1457-26-3$ is the second tangled diagram of (1.2). In the standard representation of a partition any vertex of degree two, say $j$, is incident to the noncrossing $\operatorname{arcs}(i, j)$ and $(j, s)$, where $i<j<s$.
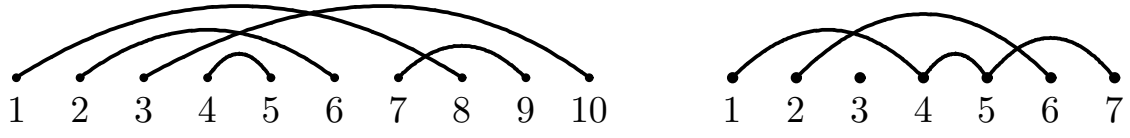

Chen et al. observed that there is a bijection between vacillating tableaux and partitions [3]. In addition they studied enhanced partitions via hesitating tableaux. For a partition $P$, the enhanced standard representation is defined as the union of the standard representation and the set of loops $\{(i, i) \mid i$ is isolated in $P\}$. Furthermore an enhanced $k$-crossing of $P$ is a set of $k$ edges $\left(i_{1}, j_{1}\right), \ldots,\left(i_{k}, j_{k}\right)$ of the enhanced representation of $P$ such that $i_{1}<i_{2}<\cdots<i_{k} \leq j_{1}<j_{2}<\cdots<j_{k}$. Via tangled diagrams we shall integrate the concepts of vacillating and hesitating tableaux. A generalized vacillating tableaux $V_{\lambda}^{2 n}$ of shape $\lambda$ and length $2 n$ is a sequence $\left(\lambda^{0}, \lambda^{1}, \ldots, \lambda^{2 n}\right)$ of shapes with certain properties. We have $\lambda^{0}=\varnothing, \lambda^{2 n}=\lambda$ and $\left(\lambda^{2 i-1}, \lambda^{2 i}\right)$ is derived from $\lambda^{2 i-2}$, for $1 \leq i \leq n$, by an elementary move defined as follows: 
$(\varnothing, \varnothing)$ : do nothing twice; $(-\square, \varnothing)$ : first remove a square then do nothing; $(\varnothing,+\square)$ : first do nothing then add a square; $( \pm \square, \pm \square)$ : add/remove a square at the odd and even steps, respectively.

For instance, the sequence below is a generalized vacillating tableau:

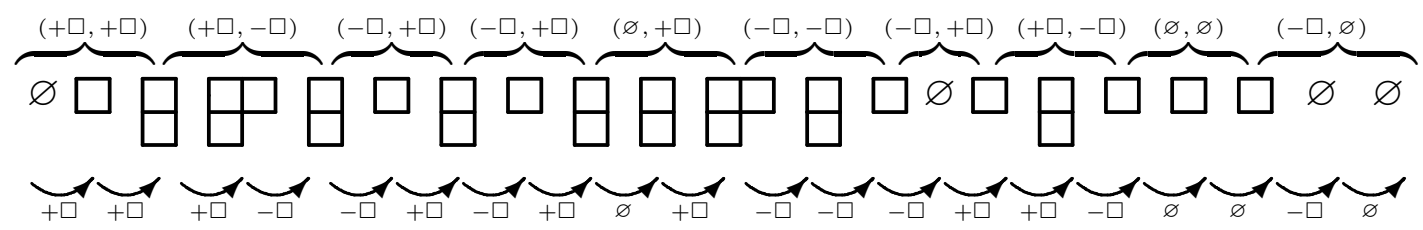

We give a bijection between $V_{\varnothing}^{2 n}$, referred to from now on as simply vacillating tableaux, and tangled diagrams on $[n]$. In fact, we show that there is a bijection between $k$ noncrossing and $k$-nonnesting tangled diagrams and we enumerate $k$-noncrossing tangled diagrams. Restricting this bijection we recover three correspondences: the bijection between vacillating tableaux with elementary moves $\{(-\square, \varnothing),(\varnothing,+\square)\}$ and matchings [3], the bijection between the vacillating tableaux with elementary moves $\{(-\square, \varnothing),(\varnothing,+\square)$, $(\varnothing, \varnothing),(-\square,+\square)\}$ and partitions and finally the bijection between the vacillating tableaux with elementary moves $\{(-\square, \varnothing),(\varnothing,+\square),(\varnothing, \varnothing),(+\square,-\square)\}$ and enhanced partitions. The latter induces a natural bijection between 2-regular $k$-noncrossing partitions and $k$-noncrossing enhanced partitions induced by contracting the arcs. This bijection is motivated by the reduction algorithm for noncrossing partitions given by Chen et al. [4].

\subsection{Motivation of tangled diagrams}

Tangled diagrams allow us to express intramolecular interactions of RNA molecules. One central problem in structural biology is that of predicting the spatial configuration of a molecule. For RNA this means to understand the configuration of the primary sequence composed by the four nucleotides $\mathbf{A}, \mathbf{G}, \mathbf{U}$ and $\mathbf{C}$. These nucleotides can form WatsonCrick (A-U, G-C) and (U-G) base pairs, as well as hydrogen bonds. The formation of these bonds stabilizes the molecular structure. Each nucleotide can form at most two chemical bonds and the latter cannot arbitrarily cross each other. Accordingly, RNA molecules form helical structures which, in many cases, determine their function. For prediction algorithms that compute for a given sequence the configuration of minimum free energy, it is of vital importance to design combinatorial frameworks which facilitate the systematic search of the configuration space. For a particular class of RNA structures, the pseudoknot RNA structures [8], partial matchings with certain arc-length conditions [7] were used to translate the biochemistry of nucleotide interactions [1] into crossings and nestings. In addition to crossings and nestings, constraints on the minimum length of bonds are typical. Tangled diagrams suit the purpose for expressing all intramolecular bonds and provide the combinatorial framework vital for efficient prediction algorithms. 


\section{Tangled diagrams and vacillating tableaux}

\subsection{Tangled diagrams}

A tangled diagram is a labeled graph, $G_{n}$, on $[n]$ with vertices of degree at most two. It is represented by drawing its vertices on a horizontal line and its $\operatorname{arcs}(i, j)$ in the upper halfplane having the following properties: two $\operatorname{arcs}\left(i_{1}, j_{1}\right)$ and $\left(i_{2}, j_{2}\right)$ are crossing if $i_{1}<i_{2}<j_{1}<j_{2},\left(i_{1}, j_{1}\right)$ and $\left(i_{2}, j_{2}\right)$ are nesting if $i_{1}<i_{2}<j_{2}<j_{1}$. Two $\operatorname{arcs}\left(i, j_{1}\right)$ and $\left(i, j_{2}\right)$ and $j_{1}<j_{2}$ (with common left-endpoint) can be drawn in two ways: if $\left(i, j_{1}\right)$ is drawn strictly below $\left(i, j_{2}\right)$ then $\left(i, j_{1}\right)$ and $\left(i, j_{2}\right)$ are called nesting (at $\left.i\right)$ and otherwise we call $\left(i, j_{1}\right)$ and $\left(i, j_{2}\right)$ crossing:

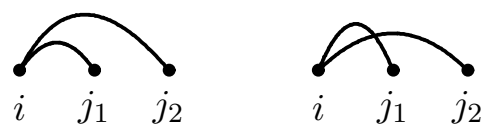

The case of two $\operatorname{arcs}\left(i_{1}, j\right),\left(i_{2}, j\right)$, where $i_{1}<i_{2}$ (with common right-endpoint) is given by:

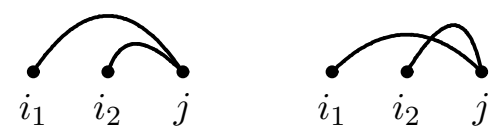

In case of a pair of arcs with common endpoints $i$ and $j$, we have:
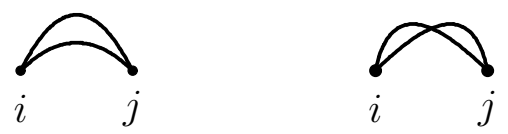

Suppose that $i<j<h$ and that we are given two $\operatorname{arcs}(i, j)$ and $(j, h)$. Then we can draw them intersecting once or not. In the former case $(i, j)$ and $(j, h)$ are crossing:
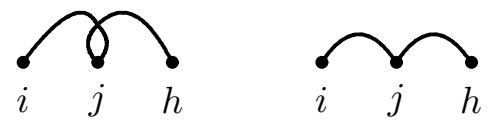

The set of all tangled diagrams on $[n]$ is denoted by $\mathcal{G}_{n}$. A tangled diagram is $k$-noncrossing if it does not contain any $k$ mutually crossing arcs and $k$-nonnesting if it does not contain any $k$ mutually nesting arcs.

\subsection{Inflation}

In this section we introduce the "local" inflation of a tangled diagram. Intuitively, a tangled diagram with $\ell$ vertices of degree two is expanded into a partial matching on $n+\ell$ vertices. For this purpose, we consider the following linear ordering on $\left\{1,1^{\prime}, \ldots, n, n^{\prime}\right\}$

$$
1<1^{\prime}<2<2^{\prime}<\cdots<n<n^{\prime} .
$$


Let $G_{n}$ be a tangled diagram with exactly $\ell$ vertices of degree two. Then the inflation of $G_{n}, \eta\left(G_{n}\right)$, is a labeled graph on $\{1, \ldots, n+\ell\}$ vertices with degree less or equal to one, obtained as follows:

Suppose first we have $i<j_{1}<j_{2}$. If $\left(i, j_{1}\right),\left(i, j_{2}\right)$ are crossing, then we map $\left(\left(i, j_{1}\right),\left(i, j_{2}\right)\right)$ into $\left(\left(i, j_{1}\right),\left(i^{\prime}, j_{2}\right)\right)$ and if $\left(i, j_{1}\right),\left(i, j_{2}\right)$ are nesting then $\left(\left(i, j_{1}\right),\left(i, j_{2}\right)\right)$ is mapped into $\left(\left(i, j_{2}\right),\left(i^{\prime}, j_{1}\right)\right)$. That is, we have the following situation:

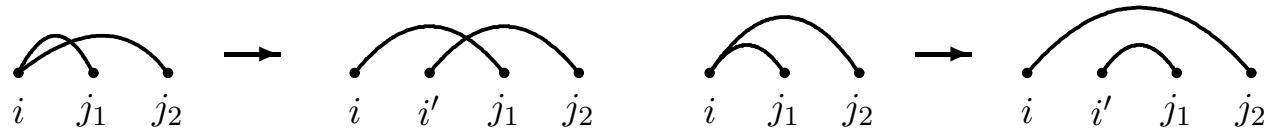

Second let $i_{1}<i_{2}<j$. If $\left(i_{1}, j\right),\left(i_{2}, j\right)$ are crossing then we map $\left(\left(i_{1}, j\right),\left(i_{2}, j\right)\right)$ into $\left(\left(i_{1}, j\right),\left(i_{2}, j^{\prime}\right)\right)$. If $\left(i_{1}, j\right),\left(i_{2}, j\right)$ are nesting then we map $\left(\left(i_{1}, j\right),\left(i_{2}, j\right)\right)$ into $\left(\left(i_{1}, j^{\prime}\right),\left(i_{2}, j\right)\right)$, i.e.:

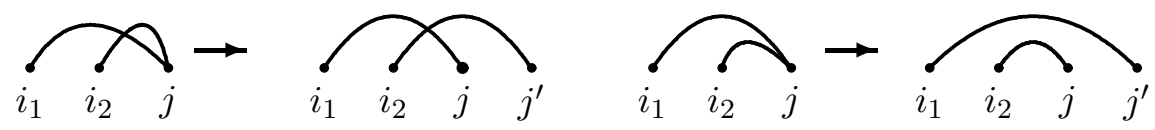

Suppose next we have $i<j$. If $(i, j),(i, j)$ are crossing arcs, then $((i, j),(i, j))$ is mapped into $\left((i, j),\left(i^{\prime}, j^{\prime}\right)\right)$ and if $(i, j),(i, j)$ are nesting arcs, then we map $((i, j),(i, j))$ into $\left(\left(i, j^{\prime}\right),\left(i^{\prime}, j\right)\right)$. Finally, if $(i, i)$ is a loop we map $(i, i)$ into $\left(i, i^{\prime}\right)$ :

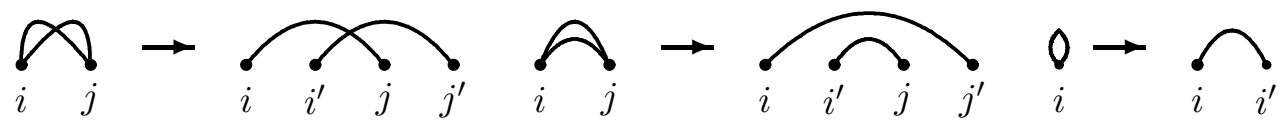

Finally, suppose we have $i<j<h$. If $(i, j),(j, h)$ are crossing, then we map $((i, j),(j, h))$ into $\left(\left(i, j^{\prime}\right),\left(j^{\prime}, h\right)\right)$ and we map $((i, j),(j, h))$ into $\left((i, j),\left(j^{\prime}, h\right)\right)$, otherwise. That is we have the situation:

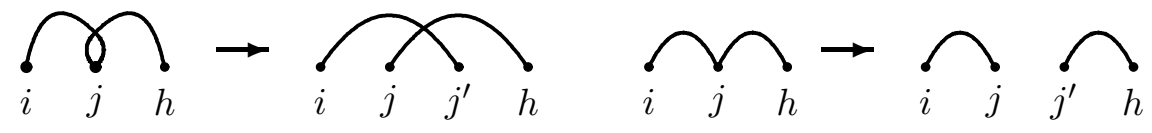

Identifying all vertex-pairs $\left(i, i^{\prime}\right)$ recovers the original tangled diagram and we have the bijection

$$
\eta: \mathcal{G}_{n} \longrightarrow \eta\left(\mathcal{G}_{n}\right)
$$

By construction, $\eta$ preserves crossings and nestings, respectively. Equivalently, a tangled diagram $G_{n}$ is $k$-noncrossing or $k$-nonnesting if and only if its inflation $\eta\left(G_{n}\right)$ is $k$-noncrossing or $k$-nonnesting, respectively. For instance, the inflation of the second tangled diagram in (1.1), is given by:

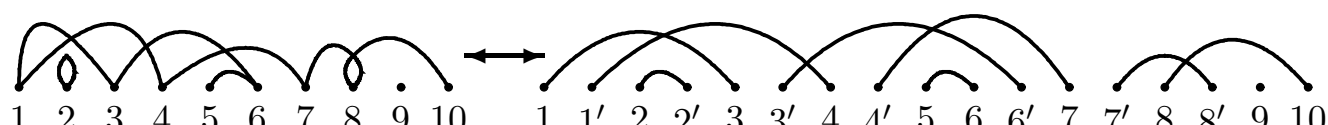




\subsection{Vacillating tableaux}

A Ferrers diagram (shape) is a collection of squares arranged in left-justified rows with weakly decreasing number of boxes in each row. A standard Young tableau (SYT) is a filling of the squares by numbers which is strictly decreasing in each row and in each column. We refer to standard Young tableaux as Young tableaux. Elements can be inserted into SYT via the RSK-algorithm [11]. We will refer to SYT simply as tableaux. The following lemma [3] is instrumental for constructing the bijection between vacillating tableaux and tangled diagrams.

Lemma 2.1. Suppose we are given two shapes $\lambda^{i} \subsetneq \lambda^{i-1}$, which differ by exactly one square. Let $T_{i-1}$ and $T_{i}$ be SYT of shape $\lambda^{i-1}$ and $\lambda^{i}$, respectively. Then there exists a unique $j$ contained in $T_{i-1}$ and a unique tableau $T_{i}$ such that $T_{i-1}$ is obtained from $T_{i}$ by inserting $j$ via the RSK-algorithm.

Proof. First, let us assume that $\lambda^{i-1}$ differs from $\lambda^{i}$ by the rightmost square in the first row. Suppose this square contains the entry $x$. Then $x$ is the unique element of $T_{i-1}$ which, if RSK-inserted into $T_{i}$, produces the tableau $T_{i-1}$. Second, suppose the square which is being removed from $\lambda^{i-1}$, is at the end of row $\ell$ and contains the entry $x$. Then we remove the square and RSK-insert $x$ into the $(\ell-1)$-th row in the square which contains $y$, such that $y$ is maximal subject to $y<x$. Therefore, if we RSK-insert $y$ into row $(\ell-1)$, it would push down $x$ in its original position. Since each column is strictly increasing, such an $y$ always exists. We conclude by induction on $\ell$ that this process results in exactly one element $j$ being removed from $T_{i-1}$ and a filling of $\lambda^{i}$, i.e. a unique tableau $T_{i}$. By construction, the RSK-insertion of $j$ recovers the tableaux $T_{i-1}$.

Below is an illustration of Lemma 2.1:

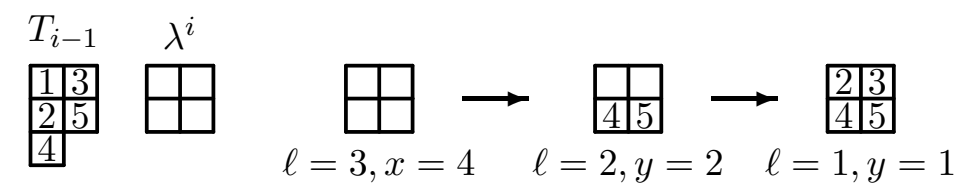

Definition 2.2. A vacillating tableau $V_{\lambda}^{2 n}$ of shape $\lambda$ and length $2 n$ is a sequence $\left(\lambda^{0}, \lambda^{1}\right.$, $\left.\ldots, \lambda^{2 n}\right)$ of shapes such that (i) $\lambda^{0}=\varnothing$ and $\lambda^{2 n}=\lambda$, and (ii) $\left(\lambda^{2 i-1}, \lambda^{2 i}\right)$ is derived from $\lambda^{2 i-2}$, for $1 \leq i \leq n$, by one of the following operations: $(\varnothing, \varnothing)$ : do nothing twice; $(-\square, \varnothing)$ : first remove a square then do nothing; $(\varnothing,+\square)$ : first do nothing then adding a square; $( \pm \square, \pm \square)$ : add/remove a square at the odd and even steps, respectively. We denote the set of such vacillating tableaux by $\mathcal{V}_{\lambda}^{2 n}$. 


\section{The bijection}

Lemma 3.1. Any vacillating tableaux of shape $\varnothing$ and length $2 n, V_{\varnothing}^{2 n}$, induces a unique inflation of some tangled diagram on $[n], \phi\left(V_{\varnothing}^{2 n}\right)$. Namely, we have the mapping

$$
\phi: V_{\varnothing}^{2 n} \longrightarrow \eta\left(\mathcal{G}_{n}\right) \text {. }
$$

Proof. In order to define $\phi$, we recursively define a sequence of triples

$$
\left(\left(P_{0}, T_{0}, V_{0}\right),\left(P_{1}, T_{1}, V_{1}\right), \ldots,\left(P_{2 n}, T_{2 n}, V_{2 n}\right)\right),
$$

where $P_{i}$ is a set of arcs, $T_{i}$ is a tableau of shape $\lambda^{i}$, and $V_{i} \subset\left\{1,1^{\prime}, 2,2^{\prime}, \ldots, n, n^{\prime}\right\}$ is a set of vertices. $P_{0}=\varnothing, T_{0}=\varnothing$ and $V_{0}=\varnothing$. We assume that the left- and right-endpoints of all $P_{i}$-arcs and the entries of the tableau $T_{i}$ are contained in $\left\{1,1^{\prime}, \ldots, n, n^{\prime}\right\}$. Once given $\left(P_{2 j-2}, T_{2 j-2}, V_{2 j-2}\right)$, we derive $\left(P_{2 j-1}, T_{2 j-1}, V_{2 j-1}\right)$ and $\left(P_{2 j}, T_{2 j}, V_{2 j}\right)$ as follows:

$(\varnothing, \varnothing)$. If $\lambda^{2 j-1}=\lambda^{2 j-2}$ and $\lambda^{2 j}=\lambda^{2 j-1}$, then we have $\left(P_{2 j-1}, T_{2 j-1}\right)=\left(P_{2 j-2}, T_{2 j-2}\right)$, ${\overline{\left(P_{2 j}, T_{2 j}\right.}}_{2}=\left(P_{2 j-1}, T_{2 j-1}\right), V_{2 j-1}=V_{2 j-2} \cup\{j\}$ and $V_{2 j}=V_{2 j-1}$.

$(-\square, \varnothing)$. If $\lambda^{2 j-1} \subsetneq \lambda^{2 j-2}$ and $\lambda^{2 j}=\lambda^{2 j-1}$, then $T_{2 j-1}$ is the unique tableau of shape $\lambda^{2 j-1}$ such that $T_{2 j-2}$ is obtained by RSK-inserting the unique number $i$ into $T_{2 j-1}, P_{2 j-1}=$ $P_{2 j-2} \cup\{(i, j)\},\left(P_{2 j}, T_{2 j}\right)=\left(P_{2 j-1}, T_{2 j-1}\right), V_{2 j-1}=V_{2 j-2} \cup\{j\}$ and $V_{2 j}=V_{2 j-1}$.

$(\varnothing,+\square)$. If $\lambda^{2 j-1}=\lambda^{2 j-2}$ and $\lambda^{2 j} \supsetneq \lambda^{2 j-1}$, then $\left(P_{2 j-1}, T_{2 j-1}\right)=\left(P_{2 j-2}, T_{2 j-2}\right), P_{2 j}=$ $\overline{P_{2 j-1} \text { and }} T_{2 j}$ is obtained from $T_{2 j-1}$ by adding the entry $j$ in the square $\lambda^{2 j} \backslash \lambda^{2 j-1}$, $V_{2 j-1}=V_{2 j-2}$ and $V_{2 j}=V_{2 j-1} \cup\{j\}$.

$(-\square,+\square)$. If $\lambda^{2 j-1} \subsetneq \lambda^{2 j-2}$ and $\lambda^{2 j} \supsetneq \lambda^{2 j-1}$, then $T_{2 j-1}$ is the unique tableau of shape

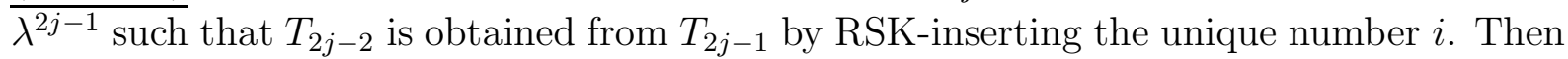
we set $P_{2 j-1}=P_{2 j-2} \cup\{(i, j)\}, P_{2 j}=P_{2 j-1}$ and $T_{2 j}$ is obtained from $T_{2 j-1}$ by adding the entry $j^{\prime}$ in the square $\lambda^{2 j} \backslash \lambda^{2 j-1}, V_{2 j-1}=V_{2 j-2} \cup\{j\}$ and $V_{2 j}=V_{2 j-1} \cup\left\{j^{\prime}\right\}$.

$(+\square,-\square)$. If $\lambda^{2 j-2} \subsetneq \lambda^{2 j-1}$ and $\lambda^{2 j} \subsetneq \lambda^{2 j-1}$, then $T_{2 j-1}$ is obtained from $T_{2 j-2}$ by adding $\overline{\text { the entry }} j$ in the square $\lambda^{2 j-1} \backslash \lambda^{2 j-2}$ and the tableau $T_{2 j}$ is the unique tableau of shape $\lambda^{2 j}$ such that $T_{2 j-1}$ is obtained from $T_{2 j}$ by RSK-inserting the unique number $i$. We then set $P_{2 j-1}=P_{2 j-2}, P_{2 j}=P_{2 j-1} \cup\left\{\left(i, j^{\prime}\right)\right\}, V_{2 j-1}=V_{2 j-2} \cup\{j\}$ and $V_{2 j}=V_{2 j-1} \cup\left\{j^{\prime}\right\}$. $(-\square,-\square)$. If $\lambda^{2 j-1} \subsetneq \lambda^{2 j-2}$ and $\lambda^{2 j} \subsetneq \lambda^{2 j-1}$, let $T_{2 j-1}$ be the unique tableau of shape

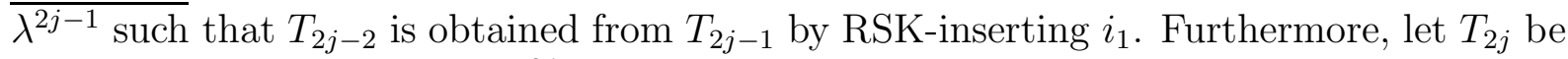
the unique tableau of shape $\lambda^{2 j}$ such that $T_{2 j-1}$ is obtained from $T_{2 j}$ by RSK-inserting $i_{2}$.

We then have $P_{2 j-1}=P_{2 j-2} \cup\left\{\left(i_{1}, j\right)\right\}, P_{2 j}=P_{2 j-1} \cup\left\{\left(i_{2}, j^{\prime}\right)\right\}, V_{2 j-1}=V_{2 j-2} \cup\{j\}$ and $V_{2 j}=V_{2 j-1} \cup\left\{j^{\prime}\right\}$.

$(+\square,+\square)$. If $\lambda^{2 j-1} \supsetneq \lambda^{2 j-2}$ and $\lambda^{2 j} \supsetneq \lambda^{2 j-1}$, we set $P_{2 j-1}=P_{2 j-2}$, and $T_{2 j-1}$ is obtained $\overline{\text { from } T_{2 j-2}}$ by adding the entry $j$ in the square $\lambda^{2 j-1} \backslash \lambda^{2 j-2}$. Furthermore we set $P_{2 j}=$ $P_{2 j-1}$ and $T_{2 j}$ is obtained from $T_{2 j-1}$ by adding the entry $j^{\prime}$ in the square $\lambda^{2 j} \backslash \lambda^{2 j-1}$, $V_{2 j-1}=V_{2 j-2} \cup\{j\}$ and $V_{2 j}=V_{2 j-1} \cup\left\{j^{\prime}\right\}$.

Claim. The image $\phi\left(V_{\varnothing}^{2 n}\right)$ is the inflation of a tangled diagram.

First, if $(i, j) \in P_{2 n}$, then $i<j$. Second, any vertex $j$ can occur only as either a left- or right-endpoint of an arc, whence $\phi\left(V_{\varnothing}^{2 n}\right)$ is a 1-diagram. Each step $(+\square,+\square)$ induces a pair of arcs of the form $\left(i, j_{1}\right),\left(i^{\prime}, j_{2}\right)$ and each step $(-\square,-\square)$ induces a pair of arcs of 
the form $\left(i_{1}, j\right),\left(i_{2}, j^{\prime}\right)$. Each step $(-\square,+\square)$ corresponds to a pair of arcs $(h, j),\left(j^{\prime}, s\right)$ where $h<j<j^{\prime}<s$, and each step $(+\square,-\square)$ induces a pair of arcs of the form $(j, s)$, $\left(h, j^{\prime}\right)$, where $h<j<j^{\prime}<s$ or a 1 -arc of the form $\left(i, i^{\prime}\right)$. Let $\ell$ be the number of steps not containing $\varnothing$. By construction each of these steps adds the 2 -set $\left\{j, j^{\prime}\right\}$, whence $\left(V_{2 n}, P_{2 n}\right)$ corresponds to the inflation of a unique tangled diagram with $\ell$ vertices of degree two and the claim follows.

Remark 3.2. The mapping $\phi$ : if squares are added, then the corresponding numbers are inserted. If squares are deleted, Lemma 2.1 is used to extract a unique number, which then forms the left-endpoint of the derived arcs.

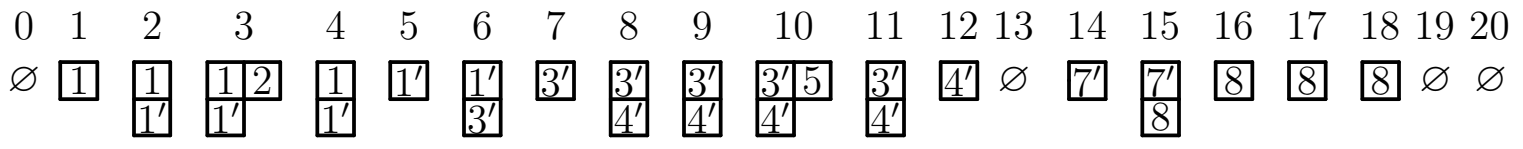

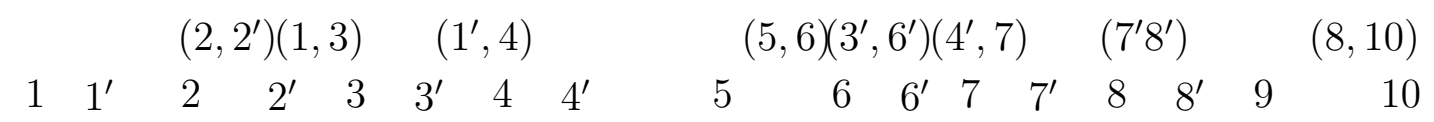

Remark 3.3. As an illustration of the mapping $\phi: V_{\varnothing}^{2 n} \longrightarrow \eta\left(\mathcal{G}_{n}\right)$, we display all arcconfigurations of inflated tangled diagrams induced by the vacillating tableaux.
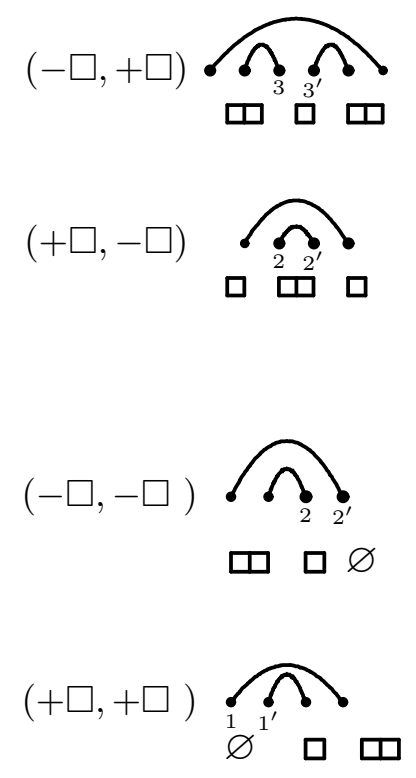
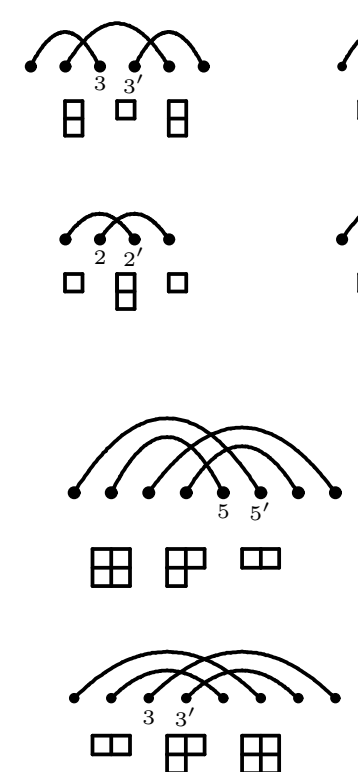
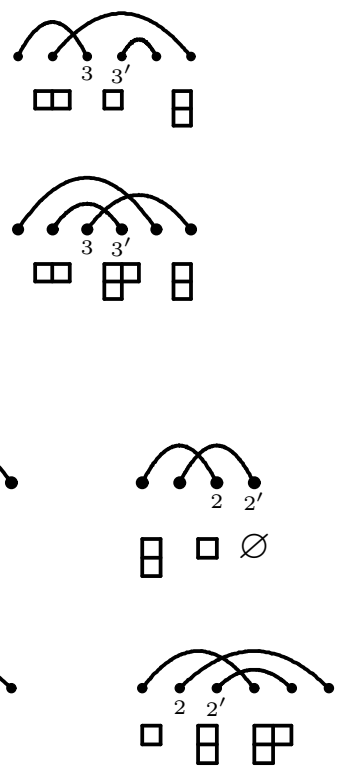
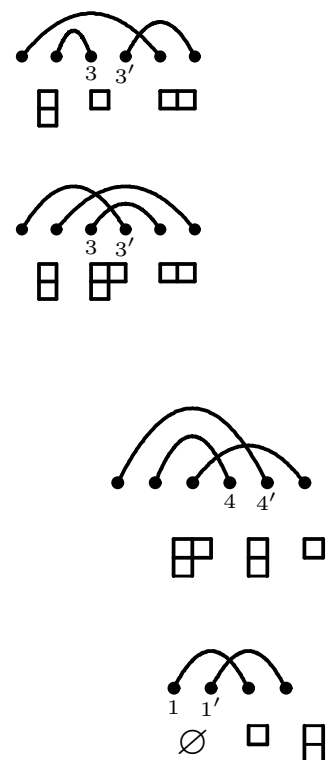

We proceed to construct the inverse of $\phi$.

Lemma 3.4. Any inflation of a tangled diagram on $n$ vertices, $\eta\left(G_{n}\right)$, induces the vacillating tableaux of shape $\varnothing$ and length $2 n, \psi\left(\eta\left(G_{n}\right)\right)$. Namely, we have the mapping

$$
\psi: \eta\left(\mathcal{G}_{n}\right) \longrightarrow \mathcal{V}_{\varnothing}^{2 n}
$$


Proof. We define $\psi$ as follows. Let $\eta\left(G_{n}\right)$ be the inflation of the tangled diagram $G_{n}$. We set

$$
\eta_{i}= \begin{cases}\left(i, i^{\prime}\right), & \text { iff } i \text { has degree two in } G_{n}, \\ i, & \text { otherwise. }\end{cases}
$$

Let $T_{2 n}=\varnothing$ be the empty tableau. We will construct a sequence of tableaux $T_{h}$ of shape $\lambda_{\eta\left(G_{n}\right)}^{h}$, where $h \in\{0,1, \ldots 2 n\}$ by considering $\eta_{i}$ for $i=n, n-1, n-2, \ldots, 1$. For each $\eta_{j}$ we inductively define the pair of tableaux $\left(T_{2 j}, T_{2 j-1}\right)$ :

(I) $\eta_{j}=j$ is an isolated vertex in $\eta\left(G_{n}\right)$, then we set $T_{2 j-1}=T_{2 j}$ and $T_{2 j-2}=T_{2 j-1}$. Accordingly, $\lambda_{\eta\left(G_{n}\right)}^{2 j-1}=\lambda_{\eta\left(G_{n}\right)}^{2 j}$ and $\lambda_{\eta\left(G_{n}\right)}^{2 j-2}=\lambda_{\eta\left(G_{n}\right)}^{2 j-1}$ (left to right: $(\varnothing, \varnothing)$ ).

(II) $\eta_{j}=j$ is the right-endpoint of exactly one arc $(i, j)$ but not a left-endpoint, then we set $T_{2 j-1}=T_{2 j}$ and obtain $T_{2 j-2}$ by RSK-inserting $i$ into $T_{2 j-1}$. Consequently, we have $\lambda_{\eta\left(G_{n}\right)}^{2 j-1}=\lambda_{\eta\left(G_{n}\right)}^{2 j}$ and $\lambda_{\eta\left(G_{n}\right)}^{2 j-2} \supsetneq \lambda_{\eta\left(G_{n}\right)}^{2 j-1}$ (left to right: $(-\square, \varnothing)$ ).

(III) $j$ is the left-endpoint of exactly one arc $(j, k)$ but not a right-endpoint, then first set $T_{2 j-1}$ to be the tableau obtained by removing the square with entry $j$ from $T_{2 j}$ and let $T_{2 j-2}=T_{2 j-1}$. Therefore $\lambda_{\eta\left(G_{n}\right)}^{2 j-1} \subsetneq \lambda_{\eta\left(G_{n}\right)}^{2 j}$ and $\lambda_{\eta\left(G_{n}\right)}^{2 j-2}=\lambda_{\eta\left(G_{n}\right)}^{2 j-1}$ (left to right: $(\varnothing,+\square)$ ).

(IV) $j$ is a left- and right-endpoint, then we have the two $\eta\left(G_{n}\right)$-arcs $(i, j)$ and $\left(j^{\prime}, h\right)$, where $i<j<j^{\prime}<h$. First the tableaux $T_{2 j-1}$ is obtained by removing the square with entry $j^{\prime}$ in $T_{2 j}$. Second the RSK-insertion of $i$ into $T_{2 j-1}$ generates the tableau $T_{2 j-2}$. Accordingly, we derive the shapes $\lambda_{\eta\left(G_{n}\right)}^{2 j-1} \subsetneq \lambda_{\eta\left(G_{n}\right)}^{2 j}$ and $\lambda_{\eta\left(G_{n}\right)}^{2 j-2} \supsetneq \lambda_{\eta\left(G_{n}\right)}^{2 j-1}$ (left to right: $(-\square,+\square)$ ).

$(\mathrm{V}) j$ is a right-endpoint of degree two, then we have the two $\eta\left(G_{n}\right)$-arcs $(i, j)$ and $\left(h, j^{\prime}\right)$. $T_{2 j-1}$ is obtained by RSK-inserting $h$ into $T_{2 j}$ and $T_{2 j-2}$ is obtained by RSK-inserting $i$ into $T_{2 j-1}$. We derive $\lambda_{\eta\left(G_{n}\right)}^{2 j-1} \supsetneq \lambda_{\eta\left(G_{n}\right)}^{2 j}$ and $\lambda_{\eta\left(G_{n}\right)}^{2 j-2} \supsetneq \lambda_{\eta\left(G_{n}\right)}^{2 j-1}$ (left to right: $(-\square,-\square)$ ).

(VI) $j$ is a left-endpoint of degree two, then we have the two $\eta\left(G_{n}\right)$-arcs $(j, r)$ and $\left(j^{\prime}, h\right)$. $T_{2 j-1}$ is obtained by removing the square with entry $j^{\prime}$ from the tableau $T_{2 j}$ and $T_{2 j-2}$ is obtained by removing the square with entry $j$ from the $T_{2 j-1}$. Then we have $\lambda_{\eta\left(G_{n}\right)}^{2 j-1} \subsetneq$ $\lambda_{\eta\left(G_{n}\right)}^{2 j}$ and $\lambda_{\eta\left(G_{n}\right)}^{2 j-2} \subsetneq \lambda_{\eta\left(G_{n}\right)}^{2 j-1}$ (left to right: $(+\square,+\square)$ ).

(VII) $j$ is a left- and right-endpoint of crossing arcs or a loop, then we have the two $\eta\left(G_{n}\right)$-arcs $(j, s)$ and $\left(h, j^{\prime}\right), h<j<j^{\prime}<s$ or an arc of the form $\left(j, j^{\prime}\right) . T_{2 j-1}$ is obtained by RSK-inserting $h$ (or $j$ in case of $\left(j, j^{\prime}\right)$ ) into the tableau $T_{2 j}$ and $T_{2 j-2}$ is obtained by removing the square with entry $j$ (or $j$ again, in case of $\left(j, j^{\prime}\right)$ ) from the $T_{2 j-1}$ (left to right: $(+\square,-\square)$ ).

Therefore, $\psi$ maps the inflation of a tangled diagram into a vacillating tableau and the lemma follows.

Remark 3.5. An illustration of Lemma 3.4: starting from right to left the vacillating tableaux is obtained via the RSK-algorithm as follows: if $j$ is a right-endpoint, it gives rise to the RSK-insertion of its (unique) left-endpoint, and if $j$ is a left-endpoint the square 
filled with $j$ is removed.

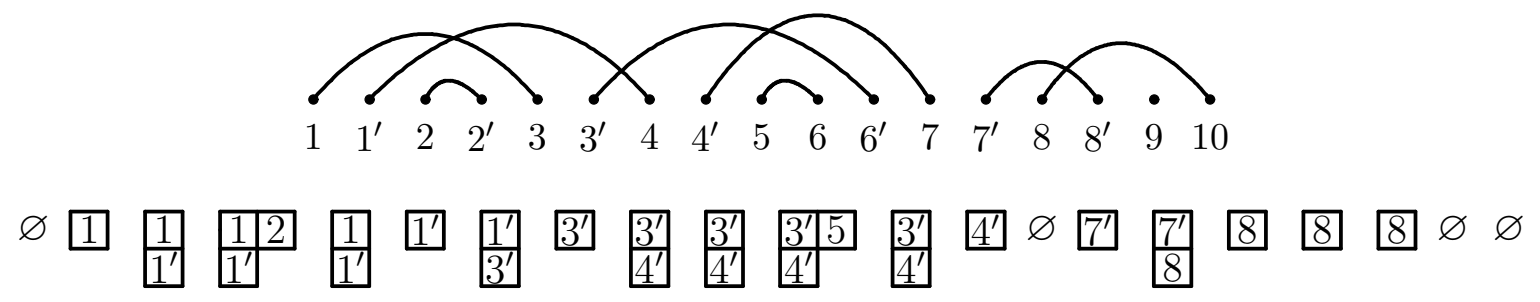

Theorem 3.6. There exists a bijection between the set of vacillating tableaux of shape $\varnothing$ and length $2 n, \mathcal{V}_{\varnothing}^{2 n}$, and the set of tangled diagrams on $n$ vertices, $\mathcal{G}_{n}$,

$$
\beta: \mathcal{V}_{\varnothing}^{2 n} \longrightarrow \mathcal{G}_{n}
$$

Proof. According to Lemma 3.1 and Lemma 3.4, we have the mappings $\phi: \mathcal{V}_{\varnothing}^{2 n} \longrightarrow \eta\left(\mathcal{G}_{n}\right)$ and $\psi: \eta\left(\mathcal{G}_{n}\right) \longrightarrow \mathcal{V}_{\varnothing}^{2 n}$. We next show that $\phi$ and $\psi$ are indeed inverses of each other. By definition, the mapping $\phi$ generates arcs whose left-endpoints, when RSK-inserted into $T_{i}$, recover the tableaux $T_{i-1}$. We observe that by definition, the mapping $\psi$ reverses this extraction: it is constructed via the RSK-insertion of the left-endpoints. Therefore we have the following relations

$$
\phi \circ \psi\left(\eta\left(G_{n}\right)\right)=\phi\left(\left(\lambda_{\eta\left(G_{n}\right)}^{h}\right)_{0}^{2 n}\right)=\eta\left(G_{n}\right) \quad \text { and } \quad \psi \circ \phi\left(V_{\varnothing}^{2 n}\right)=V_{\varnothing}^{2 n},
$$

from which we conclude that $\phi$ and $\psi$ are bijective. Since $G_{n}$ is in one to one correspondence with $\eta\left(G_{n}\right)$, the proof of the theorem is complete.

By construction, the bijection $\eta: \mathcal{G}_{n} \longrightarrow \eta\left(\mathcal{G}_{n}\right)$ preserves the maximal number of crossing and nesting arcs, respectively. Equivalently, a tangled diagram $G_{n}$ is $k$-noncrossing or $k$-nonnesting if and only if its inflation $\eta\left(G_{n}\right)$ is $k$-noncrossing or $k$-nonnesting [3]. Indeed, this follows immediately from the definition of the inflation.

Theorem 3.7. A tangled diagram $G_{n}$ is k-noncrossing if and only if all shapes $\lambda^{i}$ in the corresponding vacillating tableau have less than $k$ rows, i.e. $\phi: \mathcal{V}_{\varnothing}^{2 n} \longrightarrow \mathcal{G}_{n}$ maps vacillating tableaux having less than $k$ rows into $k$-noncrossing tangled diagrams. Furthermore, there is a bijection between the set of $k$-noncrossing and $k$-nonnesting tangled diagrams.

Theorem 3.7 is the generalization of the corresponding result in [3] to tangled diagrams. Since the inflation map enables us to interpret a tangled diagram with $\ell$ vertices of degree two on $n$ vertices as a partial matching over $n+\ell$ vertices, the proof is analogous.

We next observe that restricting the steps for vacillating tableaux leads to the bijections of Chen et al. [3]. Let $\mathcal{M}_{k}(n), \mathcal{P}_{k}(n)$ and $\mathcal{B}_{k}(n)$ denote the set of $k$-noncrossing matchings, partitions and braids, respectively. If a vacillating tableaux $V_{\varnothing}^{2 n}$ is obtained via certain steps $s \in S$ we write $V_{\varnothing}^{2 n} \models S$. 
Corollary 3.8. Let $\beta_{i}$ denote the restriction of the bijection $\beta: \mathcal{V}_{\varnothing}^{2 n} \longrightarrow \mathcal{G}_{n}$ in Theorem 3.6. Then $\beta$ induces the bijections

$$
\begin{gathered}
\beta_{1}:\left\{V_{\varnothing}^{2 n} \mid V_{\varnothing}^{2 n} \models(-\square, \varnothing),(\varnothing,+\square) \text { and has } \leq k \operatorname{rows}\right\} \rightarrow \mathcal{M}_{k}(n), \\
\beta_{2}:\left\{V_{\varnothing}^{2 n} \mid V_{\varnothing}^{2 n} \models(-\square, \varnothing),(\varnothing,+\square),(\varnothing, \varnothing),(-\square,+\square) \text { and has } \leq k \text { rows }\right\} \rightarrow \mathcal{P}_{k}(n), \\
\beta_{3}:\left\{V_{\varnothing}^{2 n} \mid V_{\varnothing}^{2 n} \models(-\square, \varnothing),(\varnothing,+\square),(\varnothing, \varnothing),(+\square,-\square) \text { and has } \leq k \text { rows }\right\} \rightarrow \mathcal{B}_{k}(n) .
\end{gathered}
$$

Remark 3.9. Corollary 3.8 implies a bijection between $k$-noncrossing braids without isolated points on [n], denoted by $\mathcal{B}_{k}^{\dagger}(n)$, and enhanced partitions on [n], see [3].

For partitions, we can illustrate the correspondence between the elementary steps and the associated tangled diagram arc-configurations as follows:
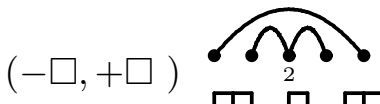

$\square \square \square$

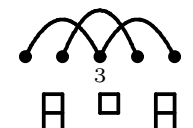

日 $\mathrm{B}$
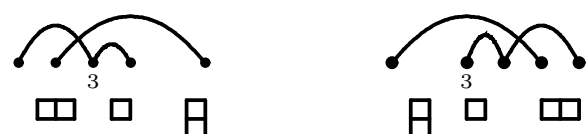

For braids, we derive the following correspondences:<smiles>[Te][Te]</smiles>

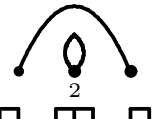

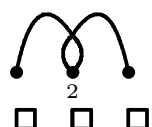

$\square$
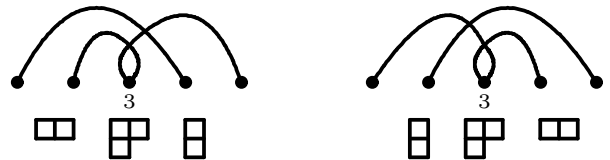

Let $t_{k}(n)$ and $\tilde{t}_{k}(n)$ be the numbers of $k$-noncrossing tangled diagrams and $k$-nonnesting tangled diagrams without isolated points on $[n]$, respectively. Furthermore, let $f_{k}(2 n-\ell)$ be the number of $k$-noncrossing matchings on $2 n-\ell$ vertices. We show that the enumeration of tangled diagrams can be reduced to the enumeration of matchings via the inflation map. Without loss of generality we can restrict our analysis to the case of tangled diagrams without isolated points since the number of tangled diagrams on $[n]$ is given by

$$
t_{k}(n)=\sum_{i=0}^{n}\left(\begin{array}{c}
n \\
i
\end{array}\right) \tilde{t}_{k}(n-i) .
$$

Theorem 3.10. The number of k-noncrossing tangled diagrams without isolated points on $[n]$ is given by

$$
\tilde{t}_{k}(n)=\sum_{\ell=0}^{n}\left(\begin{array}{l}
n \\
\ell
\end{array}\right) f_{k}(2 n-\ell),
$$

and in particular for $k=3$ we have

$$
\tilde{t}_{3}(n)=\sum_{\ell=0}^{n}\left(\begin{array}{l}
n \\
\ell
\end{array}\right)\left(C_{\frac{2 n-\ell}{2}} C_{\frac{2 n-\ell}{2}+2}-C_{\frac{2 n-\ell}{2}+1}^{2}\right) .
$$


Proof. Let $\tilde{\mathcal{T}}_{k}(n, V)$ denote the set of tangled diagrams without isolated points in which $V=\left\{i_{1}, \ldots, i_{h}\right\}$ is the set of vertices of degree one (where $h \equiv 0 \bmod 2$ by the definition of $\left.\tilde{\mathcal{T}}_{k}(n, V)\right)$ and let $\mathcal{M}_{k}\left(\left\{1,1^{\prime}, \ldots, n, n^{\prime}\right\} \backslash V^{\prime}\right)$, where $V^{\prime}=\left\{i_{1}^{\prime}, \ldots, i_{h}^{\prime}\right\}$, denotes the set of matchings on $\left\{1,1^{\prime}, \ldots, n, n^{\prime}\right\} \backslash V^{\prime}$. By construction, the inflation $\eta: \mathcal{G}_{n} \longrightarrow \eta\left(\mathcal{G}_{n}\right)$ induces a well-defined mapping

$$
\hat{\eta}: \tilde{\mathcal{T}}_{k}(n, V) \longrightarrow \mathcal{M}_{k}\left(\left\{1,1^{\prime}, \ldots, n, n^{\prime}\right\} \backslash V^{\prime}\right)
$$

with the inverse map defined by identifying all pairs $\left(x, x^{\prime}\right)$, where $x, x^{\prime} \in\left\{1,1^{\prime}, \ldots, n, n^{\prime}\right\} \backslash$ $V^{\prime}$. Obviously, we have $\left|\mathcal{M}_{k}\left(\left\{1,1^{\prime}, \ldots, n, n^{\prime}\right\} \backslash V^{\prime}\right)\right|=f_{k}(2 n-h)$ and

$$
\tilde{t}_{k}(n)=\sum_{V \subset[n]} \tilde{t}_{k}(n, V)=\sum_{\ell=0}^{n}\left(\begin{array}{l}
n \\
\ell
\end{array}\right) f_{k}(2 n-\ell) .
$$

Suppose $n \equiv 0 \bmod 2$. Let $C_{m}$ denote the $m$-th Catalan number. Then we have [6]

$$
f_{3}(n)=C_{\frac{n}{2}} C_{\frac{n}{2}+2}-C_{\frac{n}{2}+1}^{2} .
$$

and the theorem follows.

As an illustration of the bijection of (3.12) we consider two 4-noncrossing tangled diagrams, where $n=4$ and $V=\{1,3\}$ :

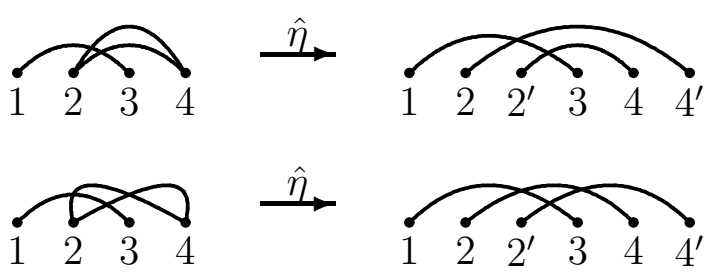

Remark 3.11. Since $f_{k}(n)$ is $D$-finite [12], Theorem 3.10 implies that $t_{k}(n)$ is $D$-finite for any $k \geq 2[10]$.

The first 10 numbers of 3-noncrossing tangled diagrams are given by:

\begin{tabular}{|c|c|c|c|c|c|c|c|c|c|c|}
\hline$n$ & 1 & 2 & 3 & 4 & 5 & 6 & 7 & 8 & 9 & 10 \\
\hline$t_{3}(n)$ & 2 & 7 & 39 & 292 & 2635 & 27019 & 304162 & 3677313 & 47036624 & 629772754 \\
\hline
\end{tabular}

The enumeration of 3-noncrossing partitions and 3-noncrossing enhanced partitions has been studied by Xin and Bousquet-Mélou [2]. The authors obtain their results by solving a functional equation of walks in the first quadrant using the reflection principle [5] and the kernel method [9].

In order to give our bijection between 2-regular $k$-noncrossing partitions and braids without isolated points, i.e. $k$-noncrossing enhanced partitions, we interpret the latter as a special class of $k$-noncrossing partitions denoted by $\mathcal{P}_{k}^{*}(n)$. This bijection is obtained 
as follows: for $\delta \in \mathcal{B}_{k}^{\dagger}(n)$, we identify loops with isolated points and crossing arcs $(i, j)$ and $(j, h)$, where $i<j<h$, by noncrossing arcs. Let $\mathcal{P}_{k, 2}(n)$ denote the set of 2-regular, $k$-noncrossing partitions, i.e. the set of $k$-noncrossing partitions without arcs of the form $(i, i+1)$.

Theorem 3.12. Let $k \in \mathbb{N}, k \geq 3$. Then we have a bijection

$$
\vartheta: \mathcal{P}_{k, 2}(n) \longrightarrow \mathcal{B}_{k}^{\dagger}(n-1), \quad \vartheta((i, j))=(i, j-1) .
$$

Proof. By construction, $\vartheta$ maps tangled diagrams on $[n]$ to tangled diagrams on $[n-1]$. Since there does not exist any arc of the form $(i, i+1)$, for any $\pi \in \mathcal{P}_{k, 2}(n), \vartheta(\pi)$ is loop-free. By construction, $\vartheta$ preserves the orientation of arcs, whence $\vartheta(\pi)$ is a partition. Claim 1. $\vartheta: \mathcal{P}_{k, 2}(n) \longrightarrow \mathcal{B}_{k}^{\dagger}(n-1)$ is well-defined.

We first prove that $\vartheta(\pi)$ is $k$-noncrossing. Suppose there exist $k$ mutually crossing arcs, $\left(i_{s}, j_{s}\right), s=1, \ldots, k$ in $\vartheta(\pi)$. Since $\vartheta(\pi)$ is a partition, we have $i_{1}<\cdots<i_{k}<j_{1}<\cdots<$ $j_{k}$. So, we obtain for the partition $\pi \in \mathcal{P}_{k, 2}(n)$ the $k \operatorname{arcs}\left(i_{s}, j_{s}+1\right), s=1, \ldots, k$ where $i_{1}<\cdots<i_{k}<j_{1}+1<\cdots<j_{k}+1$, which is impossible since $\pi$ is $k$-noncrossing. We next show that $\vartheta(\pi)$ is a $k$-noncrossing braid. If $\vartheta(\pi)$ is not a $k$-noncrossing braid, then it contains $k$ arcs of the form $\left(i_{1}, j_{1}\right), \ldots\left(i_{k}, j_{k}\right)$ such that $i_{1}<\cdots<i_{k}=j_{1}<\cdots<j_{k}$. Then $\pi$ contains the arcs $\left(i_{1}, j_{1}+1\right),\left(i_{k}, j_{k}+1\right)$ where $i_{1}<\cdots<i_{k}<j_{1}+1<\cdots<j_{k}+1$, which is impossible since these arcs are a set of $k$ mutually crossing arcs and Claim 1 follows.

Claim 2. $\vartheta$ is bijective.

Clearly $\vartheta$ is injective and it remains to prove surjectivity. For any $k$-noncrossing braid $\delta$ there exists 2-regular partition $\pi$ such that $\vartheta(\pi)=\delta$. We have to show that $\pi$ is $k$ noncrossing. Suppose that there exists some partition $\pi$ with $k$ mutually crossing arcs such that $\vartheta(\pi)=\delta$. Let $M^{\prime}=\left\{\left(i_{1}, j_{1}\right), \ldots,\left(i_{k}, j_{k}\right)\right\}$ be a set of $k$ mutually crossing arcs in the standard representation of $\pi$, i.e. $i_{1}<\cdots<i_{k}<j_{1}<\cdots<j_{k}$. Then we have in $\vartheta(\pi)$ the $\operatorname{arcs}\left(i_{s}, j_{s}-1\right), s=1, \ldots, k$ such that

$$
i_{1}<\cdots<i_{k} \leq j_{1}-1<\cdots<j_{k}-1 \text {. }
$$

Since $M=\left\{\left(i_{1}, j_{1}-1\right), \ldots,\left(i_{k}, j_{k}-1\right)\right\}$ is $k$-noncrossing, we conclude $i_{k}=j_{1}-1$. This is impossible in $k$-noncrossing braids. By transposition, we have proved that any $\vartheta$-preimage is necessarily a $k$-noncrossing partition, whence Claim 2 and the proof of the theorem is complete.

Here is an illustration of the above bijection:

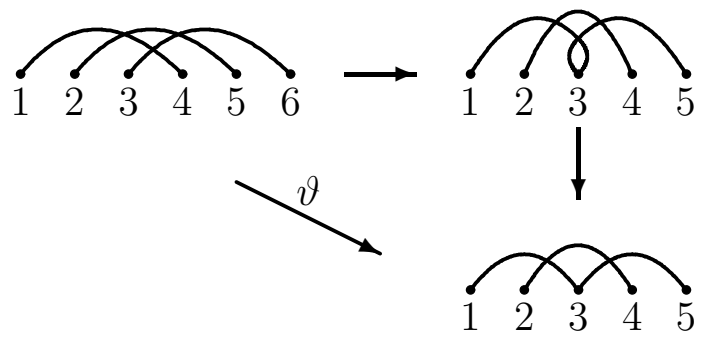


Remark 3.13. The proof of Theorem 3.12 can be generalized to a bijection between $\mathcal{P}_{k}(n)$ and $\mathcal{B}_{k}(n-1)$ for $k \geq 3$.

Acknowledgments. We are grateful to Emma Y. Jin for helpful discussions. This work was supported by the 973 Project, the PCSIRT Project of the Ministry of Education, the Ministry of Science and Technology, and the National Science Foundation of China.

\section{References}

[1] Robert T. Batey, Robert P. Rambo and Jennifer A. Doudna, Tertiary Motifs in RNA Structure and Folding, Angew. Chem. Int. Ed., 38 (1999), 2326-2343.

[2] Mireille Bousquet-Mélou and Guoce Xin, On partitions avoiding 3-crossings, Séminaire Lotharingien de Combinatoire, 54 (2006), Article B54c.

[3] William Y.C. Chen, Eva Y.P. Deng, Rosena R.X. Du, Richard P. Stanley and Catherine H. Yan, Crossings and nestings of matchings and partitions, Trans. Amer. Math. Soc., 359 (2007), No. 4, 1555-1575.

[4] William Y.C. Chen, E.Y.P. Deng and R.R.X. Du, Reduction of m-regular noncrossing partitions, Europ. J. Combin., 26 (2005), No. 2, 237-243.

[5] I.M. Gessel and D. Zeilberger, Random walk in a Weyl chamber, Proc. Amer. Math. Soc., 115 (1992), 27-31.

[6] D. Gouyou-Beauschamps, Standard Young tableaux of height 4 and 5, Europ. J. Combin., 10 (1989), 69-82.

[7] C. Haslinger and P.F. Stadler, RNA structures with pseudo-knots, Bull. Math. Biol., 61 (1999), 437-467.

[8] E.Y. Jin, J. Qin, and C.M. Reidys, Combinatorics of RNA structures with pseudoknots, Bull. Math. Biol., 70 (2008), 45-67.

[9] S. G. Mohanty, Lattice path counting and Applications, Academic Press, New York, 1979.

[10] R. P. Stanley, Differentiably finite power series, Europ. J. Combin., 1 (1980), 175-188.

[11] R. P. Stanley, Enumerative Combinatorics, vol. 1, Wadsworth and Brooks/Cole, Pacific Grove, CA, 1986; second printing, Cambridge University Press, Cambridge, 1996.

[12] R. Stanley, Enumerative Combinatorics, vol. 2, Cambridge University Press, Cambridge, 1999. 